Cite this: Phys. Chem. Chem. Phys., 2011, 13, 12240-12248

\title{
The influence of polarizability on the dielectric spectrum of the ionic liquid 1-ethyl-3-methylimidazolium triflate
}

\author{
Christian Schröder, ${ }^{a}$ Thomas Sonnleitner, $^{b}$ Richard Buchner $^{b}$ and \\ Othmar Steinhauser ${ }^{a}$
}

Received 1st March 2011, Accepted 30th April 2011

DOI: $10.1039 / \mathrm{c1cp20559e}$

This work reports for the first time the computational, frequency-dependent dielectric spectrum of the polarizable molecular ionic liquid 1-ethyl-3-methylimidazolium triflate as well as its experimental analogue. In the frequency range from $500 \mathrm{MHz}$ up to $20 \mathrm{GHz}$ the agreement between the computational and the experimental spectrum is quantitative. For higher frequencies up to $10 \mathrm{THz}$ the agreement is still remarkably good. The experimental asymptotic limit $\varepsilon_{\infty}$ is 2.3 . The difference in the computational value of 1.9 comes solely from the neglect of polarizability of the hydrogen atoms. For reasons of efficiency the simulations are based on the Lagrangian algorithm for the Drude oscillator model which cannot handle polarizable hydrogens. In the computational analysis the complete spectrum of the generalized dielectric constant $\sum_{0}^{*}(\nu)$ is splitted into its translational and non-translational components, called dielectric conductivity $\vartheta_{0}(\nu)$ and dielectric permittivity $\varepsilon(\nu)$. For 1-ethyl-3-methylimidazolium triflate both components contribute with equal weight and overlap in the complete frequency range. The inclusion of polarization forces, however, is quite different for the two components: the collective non-translational dynamics is accelerated and hence the dielectric permittivity is shifted to higher frequencies. The low frequency region of the dielectric conductivity is also affected while its high frequency part remains almost unchanged. Inductive effects are not only visible at high frequencies but also contribute in the sub-GHz region. The computational peak found in this region correlates with the experimental OKE-spectrum. It may be interpreted as the correlation between the induced dipole moment of the cations and the local electric field exerted by the anionic cage.

\section{Introduction}

Molecular ionic liquids (ILs) are an interesting class of soft matter. ${ }^{1,2}$ Their increased application as organic solvents in the past decade is not only due to their favorably low volatility but also to their unique solvent properties, e.g. the tunable hydrophobicity, polarity and viscosity, by choosing a particular combination of cations and anions., ${ }^{2,3}$ The most popular cations are imidazolium cations which consist of a heterocyclic aromatic ring with two different alkyl chains attached. Common anions are weakly basic, have a smeared-out negative charge distribution and are generally smaller than their cationic partner. ${ }^{1}$ Usually, a discussion of molecular-level factors affecting the physical properties of ILs is focused on the level of individual cations and anions, leading to general statements such as "the chemical structure of the anions has a strong influence" or "the alkyl chain length effect of imidazolium".

${ }^{a}$ University of Vienna, Department of Computational Biological Chemistry, Austria.E-mail: christian.schroeder@univie.ac.at

${ }^{b}$ University of Regensburg, Department of Physical and Theoretical Chemistry, Germany
However, as will be shown below, the situation is more complicated.

Polarity is an important solvent property as it characterizes the global solvation capability of a compound. Weingärtner et al. have shown that a wide range of molecular ILs exhibit a similar dielectric constant despite their completely different molecular structures. ${ }^{4-6}$ Also, indications for a nanostructured organization with polar and non-polar domains were found. ${ }^{7-9}$ This clearly demonstrates that a discussion of IL properties based on the mere summation of anion and cation effects is inappropriate. In fact, the outstanding behaviour of ILs arises from the complex interplay of collective rotational and translational motions in these liquids. In other words, molecular ILs are more than a simple mixture of individual anions, cations, single ions and possibly a few ion pairs.

In order to clarify the above mentioned complex interplay one may study a plethora of physical properties, either with experiments ${ }^{10-18}$ or simulations. ${ }^{19}$ However, it is reasonably expected that the combination of both approaches will give a deeper insight ${ }^{20-23}$ as on the one hand, simulations are able to decompose a physical property into contributions associated 
specifically with the cations, the anions and the coupling of both. On the other hand, the force fields used for describing the interactions of the ions in the simulations need to be validated. This is conveniently done by comparing simulated and experimental results of selected properties, like density, conductivity, viscosity or isothermal compressibility. ${ }^{24-32}$ Alternatively, spectroscopic data, ${ }^{22,33,34}$ computed properties like the heat of vaporization, ${ }^{29-32,35}$ or quantum-mechanical calculations can be used. ${ }^{36,37}$

In this work, we propose another check for the validity of a chosen computational force field: the frequency-dependent dielectric spectrum, which monitors rotational and translational motions over several orders of magnitude in frequency space. This quantity reflects the temporal evolution of IL dynamics from $\sim 100$ femtoseconds to several nanoseconds, covering processes from intra- and intermolecular vibrations via molecular rotation to the structural relaxation of large aggregates, see Fig. 2 of ref. 15 for an overview. Furthermore, various physical properties can be estimated from the dielectric spectrum. For instance, a change in viscosity is generally associated with a peak shift in the dielectric loss spectrum and the polarity of the solvent can be characterized by the zero-frequency limit of the dielectric constant. The dielectric constant also accounts for the behaviour at optical frequencies via the refractive index.

For this contribution 1-ethyl-3-methylimidazolium triflate $\left(\mathrm{EMIM}^{+} \mathrm{CF}_{3} \mathrm{SO}_{3}{ }^{-}\right)$was chosen as the target IL as both anions and cations have a dipole moment and thus should contribute to the dielectric spectrum. Individual contributions of the anions, cations and their cross-correlations to the dielectric response will be simulated and results will be compared with the experimental spectrum. A particular focus is on the influence of intramolecular (electronic) polarizability on the simulation result.

\section{Theory}

\section{A. Phenomenology of dielectric behaviour}

In an overall neutral sample exposed to a spatially homogeneous, external electric field, $\boldsymbol{E}_{0}(\omega)$, of harmonic frequency, $\omega$, a dielectric polarization, $\boldsymbol{P}(\omega)$, is induced which counteracts $\boldsymbol{E}_{0}(\omega)$. For small perturbations $\boldsymbol{P}(\omega)$ depends linearly on the internal (Maxwell) electric field $\boldsymbol{E}(\omega)$

$$
\boldsymbol{P}(\omega)=\frac{\Sigma^{*}(\omega)}{4 \pi} \boldsymbol{E}(\omega)
$$

For conducting boundary conditions, e.g. perfect Ewald summation, $\boldsymbol{E}_{0}(\omega)$ and $\boldsymbol{E}(\omega)$ are equal and may be used synonymously ${ }^{38,39}$ The susceptibility $\Sigma^{*}(\omega)$ is called "generalized dielectric constant" (GDC).

For a sample composed exclusively of neutral, polar molecules the GDC is related to the dielectric permittivity, $\varepsilon(\omega)$ (traditionally termed "dielectric constant"), via

$$
\Sigma^{*}(\omega)=\varepsilon(\omega)-1
$$

and $\boldsymbol{P}(\omega)$ is solely generated by the orientation of these neutral molecules in the external electric field, $\boldsymbol{E}_{0}(\omega)$. The term "generalized" of the GDC denotes an extension of this traditional concept to include charged species. The translation of these charged species is characterized by the conductivity, $\sigma(\omega)$, so that the total sample response to the applied field becomes ${ }^{21,40}$

$$
\begin{aligned}
\Sigma^{*}(\omega) & =\varepsilon(\omega)-1+\frac{4 \pi i}{\omega} \sigma(\omega) \\
& =\varepsilon(\omega)-1+\vartheta(\omega)
\end{aligned}
$$

The last equation decomposes the GDC into the dielectric permittivity $\varepsilon(\omega)$ and the dielectric conductivity $\vartheta(\omega) .^{21,41,42}$ The first component, $\varepsilon(\omega)$, is mainly due to dipole rotation (including intramolecular vibrations, librations and induced effects) and generally occurs in the microwave region of the dielectric spectrum, whereas $\vartheta(\omega)$ is mainly located in the far-infrared region and stems from the short-time (non-steady state) translational contribution of the charged species, $4 \pi i \sigma(\omega) / \omega$. This may include intermolecular vibrations of the ions, "cage rattling" and the translation of ion aggregates. Of course, the translation of charged species does not only give a direct contribution, $\vartheta(\omega)$, to the overall response but also changes the dielectric permittivity, $\varepsilon(\omega)$, indirectly.

The interplay or coupling between these two components depends on the molecular composition. For a simple ionic solution, e.g. $\mathrm{NaCl}$ in water, $\varepsilon(\omega)$ and $\vartheta(\omega)$ components are separated in frequency space by orders of magnitude for two reasons: first, the coupling of charge and polarity is always of intermolecular character since the charged species have no dipole moment and the dipolar molecules carry no charge. Second, the charged species have large neutral solvation shells separating them spatially and screening their interactions. Even for the case of charged proteins in water, $\varepsilon(\omega)$ and $\vartheta(\omega)$ are separated in frequency space since the viscosity shifts $\varepsilon(\omega)$ to much lower frequencies. In ILs, however, charge and dipole moment reside on the very same molecule. This implies intra- and intermolecular coupling between $\varepsilon(\omega)$ and $\vartheta(\omega)$. In other words, the contributions of $\varepsilon(\omega)$ and $\vartheta(\omega)$ to the GDC overlap, ${ }^{21,42,43}$ and this even applies when the IL is diluted by a polar solvent like water, because charged species stay in close proximity and their collective translation is visible at low frequencies. ${ }^{21}$ The important point to stress here is that it is the generalized dielectric constant, $\Sigma^{*}(\omega)$, which is measured experimentally by dielectric relaxation spectroscopy ${ }^{15,44,45}$ and which can be computed by molecular dynamics simulation. ${ }^{21,41,46-48}$

Whilst the nuclear degrees of freedom describing translation and rotation are usually explicitly considered in computer simulations, the electronic degrees are either implicitly contained in the applied force field or may be explicitly modelled by introducing (electronic) polarizability. Since in the former case electrostatic forces are restricted to the interactions between permanent charge distributions, these cannot respond to an external electric field, $\boldsymbol{E}_{0}(\omega)$, of very high frequency, $\omega$. As a result $\lim _{\omega \rightarrow \infty} \varepsilon(\omega)=1 .^{49,50}$ Modelling flexibility of the charge distributions through introduction of atomic polarizabilities enables tracking the sample response to high frequencies, so that $\lim _{\omega \rightarrow \infty} \varepsilon(\omega)=\varepsilon_{\infty}$ with $\varepsilon_{\infty}>1 .^{50-52}$ Again, the inclusion of polarizability does not only change the value of $\varepsilon_{\infty}$ but affects $\varepsilon(\omega)$ and $\vartheta(\omega)$ as well and therefore alters the positions and shapes of peaks in the simulated dielectric loss spectrum. 


\section{B. Collective dipole moments and their correlation functions}

In the computational analysis $\Sigma^{*}(\omega)$ can be evaluated from equilibrium simulations of polarizable systems by

$$
\begin{aligned}
\Sigma^{*}(\omega) & =\frac{4 \pi}{3 V} \frac{1}{k_{\mathrm{B}} T} \mathscr{L}\left[-\frac{\mathrm{d}}{\mathrm{d} t}\left\langle M_{\mathrm{tot}}(0) \cdot M_{\mathrm{tot}}(t)\right\rangle\right]+\frac{4 \pi}{3 V} \operatorname{tr}\langle\mathscr{A}\rangle \\
& =\left(\varepsilon(\omega)-\varepsilon_{\infty}\right)+\vartheta(\omega)+\left(\varepsilon_{\infty}-1\right)
\end{aligned}
$$

using linear response theory. ${ }^{21,46,50,53}$ This relation shows that for a computational analysis of the GDC the total collective dipole moment of the sample, $\boldsymbol{M}_{\text {tot }}$, is the central quantity accompanied by the macroscopic polarizability tensor, $\mathscr{A}$, which represents the electronic degrees of freedom: ${ }^{50,54}$

$$
\frac{4 \pi}{3 V} \operatorname{tr}\langle\mathscr{A}\rangle=\varepsilon_{\infty}-1
$$

In eqn (6) the high frequency limit of the GDC is only given by the last term since $\lim _{\omega \rightarrow \infty} \operatorname{Re}\left[\varepsilon(\omega)-\varepsilon_{\infty}\right]=0$ and $\lim _{\omega \rightarrow \infty} \operatorname{Re}[\vartheta(\omega)]=0$.

In order to link the phenomenology of the dielectric behaviour with the computational analysis, a decomposition of the collective dipole moment, $\boldsymbol{M}_{\text {tot }}$, into translational $\left(\boldsymbol{M}_{J}\right)$ and non-translational $\left(\boldsymbol{M}_{D}=\boldsymbol{M}_{\mathrm{tot}}-\boldsymbol{M}_{J}\right)$ dipole moments is useful, both for the interpretation as well as for technical reasons. ${ }^{55}$ In terms of autocorrelation functions this corresponds to

$$
\begin{aligned}
\Phi_{\mathrm{tot}}(t) & =\left\langle\boldsymbol{M}_{\mathrm{tot}}(0) \cdot \boldsymbol{M}_{\mathrm{tot}}(t)\right\rangle=\Phi_{D}(t)+\Phi_{J}(t) \\
\Phi_{D}(t) & =\left\langle\boldsymbol{M}_{D}(0) \cdot \boldsymbol{M}_{D}(t)\right\rangle+\left\langle\boldsymbol{M}_{D}(0) \cdot \boldsymbol{M}_{J}(t)\right\rangle \\
\Phi_{J}(t) & =\left\langle\boldsymbol{M}_{J}(0) \cdot \boldsymbol{M}_{J}(t)\right\rangle+\left\langle\boldsymbol{M}_{D}(0) \cdot \boldsymbol{M}_{J}(t)\right\rangle .
\end{aligned}
$$

In previous studies we have shown that the contribution of $\left\langle\boldsymbol{M}_{D}(0) \cdot \boldsymbol{M}_{J}(t)\right\rangle$ is almost negligible. ${ }^{46,56,57}$ As a result, the FourierLaplace transform $\mathscr{L}[\ldots]$ of the negative time derivative of $\Phi_{\text {tot }}(t)$ in eqn (6) is

$$
\mathscr{L}\left[-\frac{\mathrm{d}}{\mathrm{d} t} \Phi_{\text {tot }}(t)\right]=\left\langle\boldsymbol{M}_{D}^{2}\right\rangle+i \omega \mathscr{L}_{D D}(\omega)+\frac{i}{\omega} \mathscr{L}_{J J}(\omega)
$$

with $\mathscr{L}_{D D}(\omega)=\mathscr{L}\left[\left\langle\boldsymbol{M}_{D}(0) \cdot \boldsymbol{M}_{D}(t)\right\rangle\right]$ and $\mathscr{L}_{J J}(\omega)=\mathscr{L}[\langle\boldsymbol{J}(0) \cdot \boldsymbol{J}(t)\rangle]$. In the last two equations we have used the current $\boldsymbol{J}(t)=\mathrm{d} \boldsymbol{M}_{J}(t) / \mathrm{d} t$ representing the time derivative of the translational collective dipole moment. A detailed derivation of these formulae is given in ref. 43 and 55. Altogether, the GDC $\Sigma^{*}(\omega)$ reads

$$
\Sigma^{*}(\omega)+1=\frac{4 \pi}{3 V k_{\mathrm{B}} T}\left\{\left\langle\boldsymbol{M}_{D}^{2}\right\rangle+i \omega \mathscr{L}_{D D}(\omega)\right\}+\frac{4 \pi i}{\omega} \frac{\mathscr{L}_{J J}(\omega)}{3 V k_{\mathrm{B}} T}+\varepsilon_{\infty}
$$

$$
=\left(\varepsilon(\omega)-\varepsilon_{\infty}\right)+\vartheta(\omega)+\varepsilon_{\infty}
$$

At zero frequency the imaginary part of $\Sigma^{*}(\omega)$ diverges because of the translational part of the GDC

$$
i \mathscr{L}_{J J}(\omega)=\int_{0}^{\infty}\langle\boldsymbol{J}(0) \cdot \boldsymbol{J}(t)\rangle \frac{i \cos (\omega t)-\sin (\omega t)}{\omega} \mathrm{d} t
$$

since the limit $\lim _{\omega \rightarrow 0} \cos (\omega t) / \omega$ becomes infinity. This divergence is not found for the real part of the GDC since the zerofrequency limit of the real part of the last equation has a definite but non-zero value $\left(\lim _{\omega \rightarrow 0} \sin (\omega t) / \omega=t\right) .{ }^{41}$ Therefore, the term $4 \pi i \sigma(0) / \omega$ is subtracted from the GDC both in computational analysis

$$
\begin{aligned}
\Sigma_{0}^{*}(\omega)+1 & =\left(\varepsilon(\omega)-\varepsilon_{\infty}\right)+\frac{4 \pi i}{\omega}(\sigma(\omega)-\sigma(0))+\varepsilon_{\infty} \\
& =\left(\varepsilon(\omega)-\varepsilon_{\infty}\right)+\vartheta_{0}(\omega)+\varepsilon_{\infty}
\end{aligned}
$$

and in experiment. Because experiment cannot separately determine $\varepsilon(\omega)$ and $\vartheta_{0}(\omega)$ but can only provide the real and imaginary parts of the GDC, the notion $\Sigma_{0}^{*}(\omega)+1=\varepsilon^{\prime}(\nu)-i \varepsilon^{\prime \prime}(\nu)$, with $\nu=\omega / 2 \pi$ and $\lim _{\nu \rightarrow \infty} \varepsilon^{\prime}(\nu)=\varepsilon_{\infty}$, is common.

\section{Impact of polarizability}

In molecular dynamics simulations the polarization model introduces the flexibility of charge distributions via additional induced atomic dipole moments ${ }^{32,58-60}$

$$
\boldsymbol{\mu}_{i \beta}^{\text {ind }}(t)=\alpha_{i \beta} \cdot \boldsymbol{E}_{i \beta}(t)
$$

as a response to the local electric field, $\boldsymbol{E}_{i \beta}(t)$. The induced dipole moment, $\boldsymbol{\mu}_{i \beta}^{\text {ind }}(t)$, is still present in the absence of an external field, $\boldsymbol{E}_{0}(\omega)$. In eqn (17) $\alpha_{i \beta}$ is the polarizability of atom $\beta$ of molecule $i$ located at $\boldsymbol{r}_{i \beta}(t)$. The sum of these induced dipole moments gives the collective induced dipole moment $\boldsymbol{M}_{D}^{\text {ind }}(t)$.

For non-polarizable molecules the non-translational collective dipole moment, $\boldsymbol{M}_{D}^{\text {perm }}(t)$, contains intramolecular vibrations but refers essentially to collective rotational motion. Inclusion of polarizability in the simulations additionally yields an induced contribution, $\boldsymbol{M}_{D}^{\text {ind }}(t)$, so that the total non-translational collective dipole moment, $\boldsymbol{M}_{D}(t)$, is given by

$$
\boldsymbol{M}_{D}(t)=\boldsymbol{M}_{D}^{\text {perm }}(t)+\boldsymbol{M}_{D}^{\text {ind }}(t)
$$

In Fig. 1 the permanent and induced parts of $\boldsymbol{M}_{D}^{\text {perm }}(t)$ for EMIM ${ }^{+} \mathrm{CF}_{3} \mathrm{SO}_{3}{ }^{-}$are indicated by the red and green shaded areas, which can be computed according to

$$
\begin{aligned}
\boldsymbol{M}_{D}^{\text {perm }}(t) & =\sum_{i} \sum_{\beta} q_{i \beta}\left(\boldsymbol{r}_{i \beta}(t)-\boldsymbol{r}_{i}(t)\right) \\
\boldsymbol{M}_{D}^{\text {ind }}(t) & =\sum_{i} \sum_{\beta} \boldsymbol{\mu}_{i \beta}^{\text {ind }}(t)
\end{aligned}
$$

Here, $q_{i \beta}$ is the partial charge of atom $i \beta$ and $\boldsymbol{r}_{i}(t)$ is the centerof-mass of molecule $i$. The summation in eqn (19) and (20) runs over all molecules $i$. In principle, it may be further decomposed into contributions from different molecular types, such as cations (grey shaded area) and anions (black shaded area), by restricting the molecular summation index $i$ to cations or anions. These species-specific contributions may again be splitted into their permanent and induced parts. Of course, this implies calculation of individual autocorrelation functions, such as $\left\langle\boldsymbol{M}_{D}^{\text {ind }}{ }^{+}(0) \cdot \boldsymbol{M}_{D}^{\text {ind }}{ }^{+}(t)\right\rangle$, as well as numerous cross-correlation functions, e.g. $\left\langle\boldsymbol{M}_{D}^{\text {perm }}{ }^{+}(0) \cdot \boldsymbol{M}_{D}^{\text {perm- }}-(t)\right\rangle$. The initial value of each correlation function may be converted to a contribution to the static permittivity $\varepsilon(0)$ and displayed in a symmetric matrix. A row of this matrix shows how a molecular species couples to all other species both with respect to the permanent and the induced contribution. A typical matrix is given in Table 1 . 


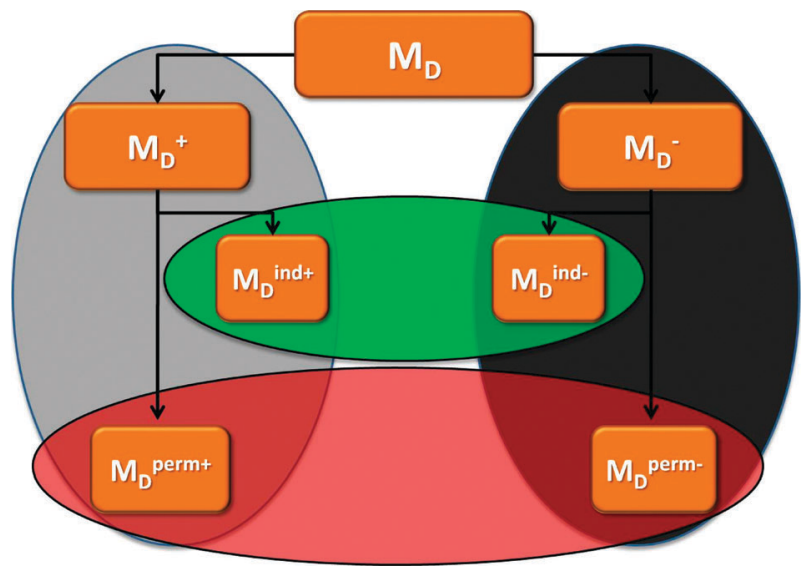

Fig. 1 Decomposition of the collective non-translational dipole moment $\boldsymbol{M}_{D}$ into cationic (gray shaded area) and anionic (black shaded area) as well as induced (green shaded area) and permanent dipole (red shaded area) contributions. The contribution from the permanent charges $\boldsymbol{M}_{D}^{\mathrm{perm}}=\sum_{i} \sum_{\beta} q_{i \beta}\left(\boldsymbol{r}_{i \beta}-\boldsymbol{r}_{i}\right)$ can be decomposed into cationic and anionic contributions by running the molecular index $i$ over cations and anions only. The contribution from the induced dipoles $\boldsymbol{M}_{D}^{\text {ind }}=\sum_{i} \sum_{\beta} \boldsymbol{\mu}_{i \beta}$ can be splitted in the same way. The color code of this figure is maintained in Fig. 2 for the cationic and anionic contributions as well as in Fig. 3 for the induced and permanent contributions.

Table 1 Contributions of correlation functions to the static permittivity $\varepsilon(0)$. The numbers in brackets represent the contribution in the case of the MD simulations with non-polarizable forces

\begin{tabular}{lllllll}
\hline & \multicolumn{2}{c}{$\mathrm{EMIM}^{+}$} & & $\mathrm{CF}_{3} \mathrm{SO}_{3}{ }^{-}$ & \\
\cline { 3 - 4 } \cline { 6 - 7 } & & Permanent & Induced & & Permanent & Induced \\
\hline $\mathrm{EMIM}^{+}$ & Permanent & $0.82(0.69)$ & 0.03 & & $0.18(0.30)$ & 0.04 \\
$\mathrm{CF}_{3} \mathrm{SO}_{3}{ }^{-}$ & Induced & 0.03 & 0.11 & & 0.36 & 0.00 \\
& Permanent & $0.18(0.30)$ & 0.36 & & $6.53(6.46)$ & -0.15 \\
& Induced & 0.04 & 0.00 & -0.15 & 0.03 \\
\hline
\end{tabular}

\section{Methods}

\section{A. Computational setup}

We performed two completely independent molecular dynamics simulations with CHARMM: ${ }^{61}$ first, we simulated 1000 $\mathrm{EMIM}^{+} \mathrm{CF}_{3} \mathrm{SO}_{3}{ }^{-}$ion pairs with a classical non-polarizable force field in a cubic box with a box length of $67.195 \AA$ under periodic boundary conditions for a simulation period of $30 \mathrm{~ns}$. The second simulation has the same setup as the first but additionally includes polarizable forces. ${ }^{54}$ For the same simulation period (30 ns) this increases the computing time by a factor of three to five.

The classical force field parameters of $\mathrm{EMIM}^{+}$were taken from ref. 26 and 62 with Lennard-Jones parameters from the AMBER force field. ${ }^{63}$ The permanent partial charges $q_{i \beta}$ were changed to the values reported in ref. 64 as done in our previous works in order to better reproduce the experimental viscosity. ${ }^{42,43,65}$ For the anions the force field parameters of ref. 66 were used without further modification. All carbonhydrogen bonds were kept fixed by the SHAKE algorithm, ${ }^{67}$ whereas all other bonds, bond angles and torsions were left flexible. The polarization of the atoms was mimicked by the so-called "Drude oscillator" model. Here, mobile Drude particles carry a charge, $q^{\delta}$, and are bound by a harmonic spring with a force constant, $k_{i \beta}^{\delta}$, to their reference atoms:

$$
k_{i \beta}^{\delta}=\frac{1}{4 \pi \varepsilon_{0}} \frac{\left(q^{\delta}\right)^{2}}{2 \alpha_{i \beta}} .
$$

The atomic polarizabilities, $\alpha_{i \beta}$, are taken from ref. 68 . The induced dipole moment, $\boldsymbol{\mu}_{i \beta}^{\text {ind }}(t)$, of atom $i \beta$ equals the distance vector, $\boldsymbol{d}_{i \beta}(t)$, between the mobile Drude particle and its reference atom times the Drude charge, $q^{\delta}$ :

$$
\boldsymbol{\mu}_{i \beta}^{\text {ind }}=q^{\delta} \cdot \boldsymbol{d}_{i \beta}(t) .
$$

For not too small $q^{\delta}$ the length of $\left\langle\boldsymbol{d}_{i \beta}\right\rangle$ decreases linearly with increasing Drude charge, making $\boldsymbol{\mu}_{i \beta}^{\text {ind }}(t)$ more or less independent of the choice of $q^{\delta}{ }^{54}$ All Drude charges in this simulations were set to $q^{\delta}=-1.0$ e. The Drude particles have a uniform mass of $m^{\delta}=0.1 \mathrm{amu}$ which is subtracted from the mass of the corresponding atom. ${ }^{69,70}$ The interactions between permanent charges $q_{i \beta}$ and Drude pairs are excluded between atoms which share a bond or an angle. ${ }^{71}$ The interactions between the corresponding Drude pairs are screened by a Thole function according to ref. 71. The Drude particles were thermostatted at $1 \mathrm{~K}$ with a relaxation time constant of $5 \mathrm{fs} .{ }^{59}$ All non-Drude particles were thermostatted at $300 \mathrm{~K}$ by a Nosé-Hoover thermostat with a relaxation time constant of 0.1 ps. In ref. 54 we have shown that these Drude parameters ensure self-consistency of the Lagrangian method.

Nonbonded and image lists were updated heuristically using a $16 \AA$ neighbour list distance. Lennard-Jones energies and forces were smoothly switched off between 11 and $12 \AA$. The electrostatic forces of both Drude particles and the attached atoms were treated by the PME technique. ${ }^{72,73}$ The "cutoff" for the real-space part interactions was $12 \AA$ with a damping constant of $0.410 \AA^{-1}$ for the reciprocal-space interactions. The grid spacing equaled $1.05 \AA$ and a sixth-order spline interpolation of the charge to the grid was used.

\section{B. Experiment}

The $\mathrm{EMIM}^{+} \mathrm{CF}_{3} \mathrm{SO}_{3}{ }^{-}$was obtained from Iolitec (Denzlingen, Germany) and dried under vacuum $\left(<10^{-8}\right.$ bar) at $\sim 40{ }^{\circ} \mathrm{C}$ for at least 7 days, yielding water contents $<200 \mathrm{ppm}$ by coulometric Karl Fischer titration. According to sample specifications halide impurity levels were $<50 \mathrm{ppm}$. The dried IL was stored in a nitrogen-filled glove box and all measurements were conducted under a dry $\mathrm{N}_{2}$ atmosphere.

Broadband dielectric spectra, $\Sigma_{0}^{*}(\nu)+1=\varepsilon^{\prime}(\nu)-i \varepsilon^{\prime \prime}(\nu)$, were obtained by combining data from a frequency-domain reflectometer based on an Agilent E8364B vector network analyzer (VNA) and Agilent 85070E-020 (0.2-20 GHz) and $85070 \mathrm{E}-050(0.5-50 \mathrm{GHz})$ probes, a wave guide interferometer covering $60-89 \mathrm{GHz}^{74}$ a transmission/reflection terahertz time-domain spectrometer (THz-TDS) at 0.3 to $3 \mathrm{THz},{ }^{75}$ and a Bruker Vertex 70 FTIR spectrometer covering the far-infrared (FIR, 0.9 to $12 \mathrm{THz}$ ) region. All measurements were conducted at $(25.00 \pm 0.05){ }^{\circ} \mathrm{C}$, except for the THz-TDS and FIR spectra, which were recorded at $(25.0 \pm 0.5){ }^{\circ} \mathrm{C}$. Details for instrument calibration and processing of the raw FIR data prior to combination with the data from the other instruments can be found in ref. 16 . 


\section{Results and discussion}

The non-translational component, $\boldsymbol{M}_{D}(t)$, of the collective dipole moment, $\boldsymbol{M}_{\mathrm{tot}}(t)$, comprises rotational, vibrational and induced contributions. Previously, we have always combined the first two contributions to $\boldsymbol{M}_{D}^{\text {perm }}(t)^{41,43,46,57,76,77}$ and the corresponding autocorrelation function, $\left\langle\boldsymbol{M}_{D}^{\text {perm }}(0) \cdot \boldsymbol{M}_{D}^{\text {perm }}(t)\right\rangle$, could be reproduced by a multi-exponential decay function, $f(t)=\sum_{k} A_{k} \exp \left(-t / \tau_{k}\right) \cdot{ }^{43}$ In the Theory section IIC of the present paper the third, induced, contribution, $\boldsymbol{M}_{D}^{\text {ind }}(t)$, was also appended to $\boldsymbol{M}_{D}(t)$ assuming that all non-translational contributions are of similar character in their time dependence.

Fig. 2 displays the individual correlation functions of the induced moment, $\left\langle\boldsymbol{M}_{D}^{\text {ind }}(0) \cdot \boldsymbol{M}_{D}^{\text {ind }}(t)\right\rangle$, for cations (grey solid line) and anions (black solid line). The inset shows the first five picoseconds, visualizing the very fast initial decay with time constants of $\tau=0.24 \mathrm{ps}$ (cations) and $\tau=0.11 \mathrm{ps}$ (anions). The exponential character of $\left\langle\boldsymbol{M}_{D}^{\text {ind }}(0) \cdot \boldsymbol{M}_{D}^{\text {ind }}(t)\right\rangle$ over the complete time span is already suggested by visual inspection of Fig. 2. Indeed, for both ions $\left\langle\boldsymbol{M}_{D}^{\text {ind }}(0) \cdot \boldsymbol{M}_{D}^{\text {ind }}(t)\right\rangle$ can be well fitted to a multi-exponential expression with the longest time constant of roughly 1000 picoseconds. This value is surprisingly long for an induced contribution and might provide an explanation for the signal at $\sim 0.6 \mathrm{GHz}$ dominating the optical Kerr-effect (OKE) spectrum of EMIM ${ }^{+} \mathrm{CF}_{3} \mathrm{SO}_{3}{ }^{-}$and other imidazolium-based ILs. ${ }^{16,78}$ In particular for the cations, the amplitude of this slow polarizability contribution accounts for almost half of the total effect. One possible explanation might be a strong correlation of the induced dipole moments, $\mu_{i \beta}^{\text {ind }}(t)$, of the cationic atoms with the field exerted by the surrounding anion cage. This might also be the reason why the cations exhibit a larger value for $\left\langle\left(\boldsymbol{M}_{D}^{\text {ind }}\right)^{2}\right\rangle$ than the anions. In any case, the cage formed by the cations around the anions is far more heterogeneous and, as a result, the local field, $\boldsymbol{E}_{i \beta}(t)$, acting on the triflate atoms is less directed. However, also for the anions induced and permanent time constants cover the same time range. Therefore, it is valid to combine permanent and induced contributions to a single quantity $\boldsymbol{M}_{D}(t)$ as illustrated in Fig. 1.

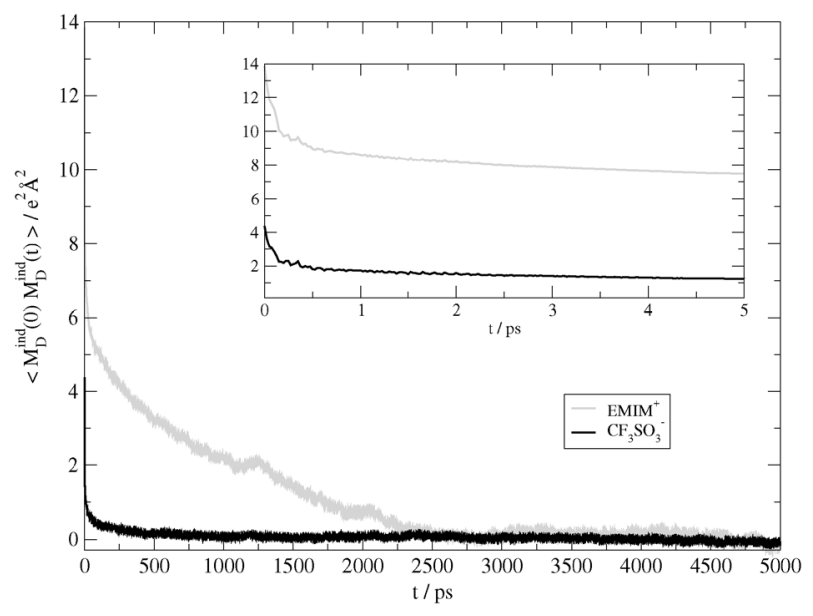

Fig. 2 Autocorrelation function $\left\langle\boldsymbol{M}_{D}^{\text {ind }}(0) \cdot \boldsymbol{M}_{D}^{\text {ind }}(t)\right\rangle$ of the collective induced dipole moments of the cations [grey] and the anions [black]. The inset depicts the first five picoseconds.

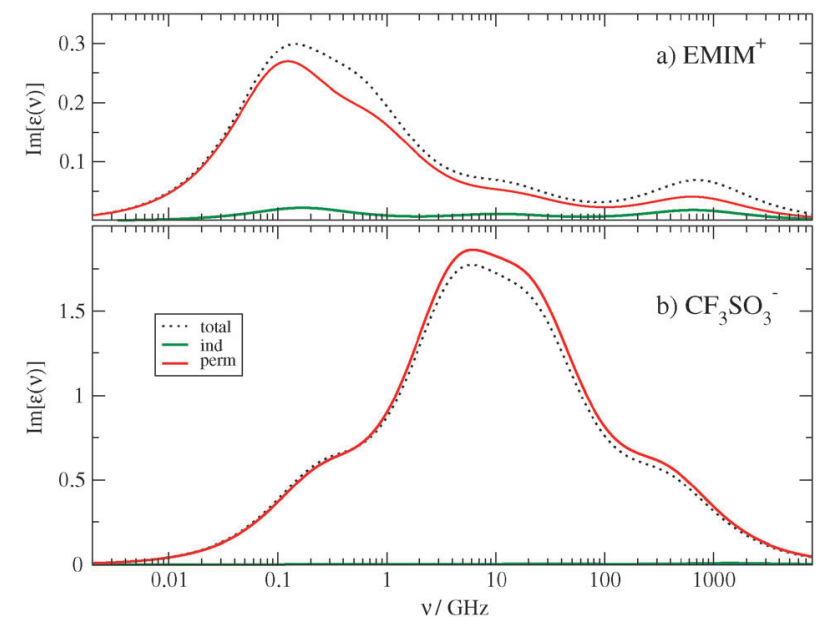

Fig. 3 Decomposition of the cationic (a) and anionic (b) contribution to the dielectric permittivity $\varepsilon(\nu)$ (dotted lines). The red and green solid lines represent the contribution from $\left\langle\boldsymbol{M}_{D}^{\text {perm }}(0) \cdot \boldsymbol{M}_{D}^{\text {perm }}(t)\right\rangle$ and $\left\langle\boldsymbol{M}_{D}^{\text {ind }}(0) \cdot \boldsymbol{M}_{D}^{\text {ind }}(t)\right\rangle$ respectively. In the case of the anions the latter contribution is negligible, but the cross-correlation $\left\langle\boldsymbol{M}_{D}^{\text {perm }}(0) \cdot \boldsymbol{M}_{D}^{\text {ind }}(t)\right\rangle$ reduces $\operatorname{Im}[\varepsilon(\nu)]$ at frequencies between 3 to $40 \mathrm{GHz}$. This reduction is also reflected in the corresponding negative contribution in Table 1. Please note the different scaling of the $y$-axes.

The slow decay of the correlation function $\left\langle\boldsymbol{M}_{D}(0) \cdot \boldsymbol{M}_{D}(t)\right\rangle$ determines the dielectric permittivity, $\varepsilon(\nu)$, which accordingly extends over a rather broad frequency range. As for $\left\langle\boldsymbol{M}_{D}(0) \cdot \boldsymbol{M}_{D}(t)\right\rangle$, we tried to separate $\varepsilon(\nu)$ into separate contributions of the cations and anions. Fig. 3 shows the obtained imaginary parts of the dielectric permittivity for the cations (a) and anions (b) with the contribution from the permanent moment, $\left\langle\boldsymbol{M}_{D}^{\text {perm }+}(0) \cdot \boldsymbol{M}_{D}^{\text {perm }+}(t)\right\rangle$ as the red lines, that of the induced dipoles, $\left\langle\boldsymbol{M}_{D}^{\text {ind }+}(0) \cdot \boldsymbol{M}_{D}^{\text {ind }+}(t)\right\rangle$, as the green lines, and the total effect as the dotted black lines. Clearly, for anions and cations the contribution from the permanent moments is much larger than that of the induced dipoles. The ratio between permanent and induced contributions, as well as their cross term, can be extracted more quantitatively from the zero-frequency value, $\varepsilon(0)$, of the corresponding real part of the dielectric permittivity. The obtained data are listed in Table 1, showing that for the cations the contributions of the permanent and induced selfterms are 0.82 and 0.11 respectively. With 0.03 the cross term between permanent and induced cationic dipoles is negligible. This supports the view that the long relaxation of the induced cation dipole, $\boldsymbol{M}_{D}^{\text {ind }}{ }^{+}(t)$, is not caused by the slow rotation of the cations but rather by the highly directing influence of the permanent charges forming the anion cage. This is reflected by the cation-anion cross term of 0.36 in Table 1 . For the anion (Fig. 3b) the permanent self-term is so dominating that the induced self-term almost vanishes. Interestingly, however, the cross term between permanent and induced dipoles is quite significant and negative, leading thus to a reduced dielectric permittivity at frequencies between 3 and $40 \mathrm{GHz}$. This reduction is also reflected in the negative value $(-0.15)$ of the corresponding $\varepsilon(0)$ in Table 1.

The accordance of the relaxation times from permanent and induced contributions leads to anion- and cation-related spectra that are both characterized by a distinct maximum and 


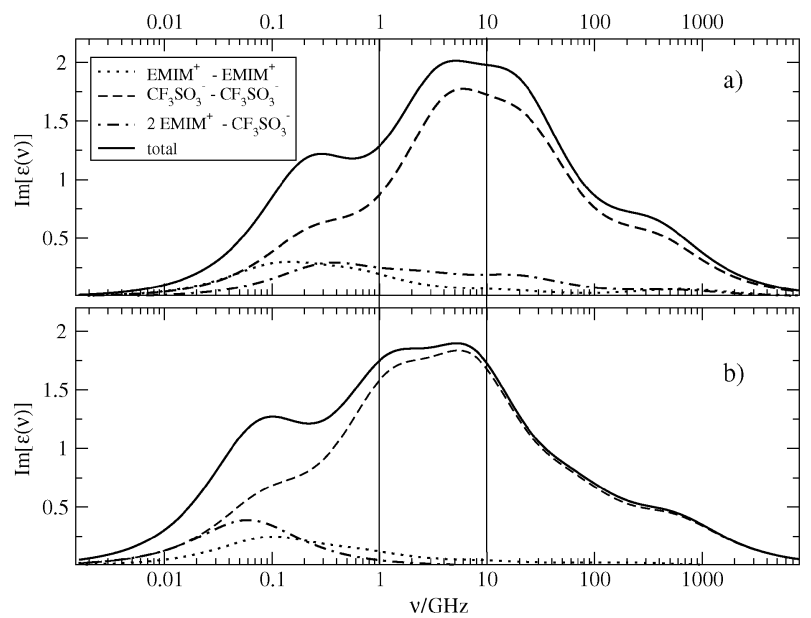

Fig. 4 Imaginary part of the dielectric permittivity $\varepsilon(\nu)$ of the polarized (a) and the non-polarized system (b).

two satellite peaks and extend $\sim 0.01 \mathrm{GHz}$ up to more than $1000 \mathrm{GHz}$. Note that the contribution arising from $\boldsymbol{M}_{D}^{\text {perm }}(t)$ is ro-vibronic, i.e. it contains librations and intramolecular vibrations which occur at high frequencies, in addition to dipole rotation dominating below $100 \mathrm{GHz}$. Superposition of cation and anion spectra (Fig. 3a and b) yields the total spectrum (Fig. 4a). Due to the overlap of the dominating cation peak and the left satellite peak of the anion a distinct peak appears in the total spectrum at $\sim 0.1 \mathrm{GHz}$, whereas the two cation-related satellites submerse under the anion contribution, which dominates the spectrum above $1 \mathrm{GHz}$.

Up to now, we have discussed the direct influence of polarizability on the dielectric spectrum arising from the induced dipole $\boldsymbol{M}_{D}^{\text {ind }}(t)$ and it was found to be rather modest (Fig. 3). However, polarizability also affects the non-inductive contributions. A first impression of this indirect effect can be gained from Table 1 where the entries for the non-polarizable system are given in brackets. For the non-polarizable system the selfterms of the anions and cations are reduced whereas the cross term is enhanced relative to the polarizable system. This shows that the inclusion of polarizability weakens the attraction between unlike species and reduces the repulsion between like species on the mesoscopic level. ${ }^{54}$ As a consequence-and this seems to be a quite general statement - inclusion of polarizability shifts all dynamical processes to higher frequencies and thus enhances the fluidity of the IL. This is clearly visible for the dielectric permittivity shown in Fig. 4a and b. Interestingly, the faster dynamics does not change the overall shape of the spectrum. The acceleration by a factor of roughly two also shows up in the diffusion coefficients and single-particle rotational constants. $^{54}$

The enhancement of fluidity also affects the dielectric conductivity, $\vartheta_{0}(\nu)$, originating from the collective translational dipole moment, $\boldsymbol{M}_{J}(t)=\sum_{i}\left(\sum_{\beta} q_{i \beta}\right) \boldsymbol{r}_{i}(t)=\sum_{i} q_{i} \boldsymbol{r}_{i}(t)$, which depends on the center-of-mass, $\boldsymbol{r}_{i}(t)$, of the molecules $i$. The role of the Drude particle for the computation of $\boldsymbol{r}_{i}(t)=\left(\sum_{\beta} m_{i \beta} \boldsymbol{r}_{i \beta}(t)+\sum_{\beta} m^{\delta} \boldsymbol{d}_{i \beta}(t)\right) / m_{i}$ is rather marginal since both the Drude mass, $m^{\delta}$, and the displacement of the mobile Drude particle, $\boldsymbol{d}_{i \beta}(t)$, are much smaller than $m_{i \beta}$ and $\boldsymbol{r}_{i \beta}(t)$. Consequently, the influence of polarizability on the dielectric conductivity is essentially of indirect nature. The frequency-dependent imaginary part of $\vartheta_{0}(\nu)$ is depicted as a dashed line for the polarizable and non-polarizable systems in Fig. 5a and b, respectively. In contrast to the dielectric permittivity, $\varepsilon(\nu)$ (shown as dotted lines), the $\vartheta_{0}$-curves are not shifted as a whole to higher frequencies when polarizability is included. Rather, the low frequency region and the high frequency part behave differently: the first main peak of $\vartheta_{0}(\nu)$ is shifted from $1 \mathrm{GHz}$ to a little bit more than $2 \mathrm{GHz}$ and now overlaps with the onset of the double-peak feature in $\varepsilon(\nu)$. Frequencies in this area correspond to relaxation time constants of roughly 60 to 100 picoseconds, which are more characteristic for the motion of molecular aggregates rather than single molecules. The overlap of $\vartheta_{0}(\nu)$ and $\varepsilon(\nu)$ suggests that the gyration of these aggregates and their translation are coupled. In the high frequency domain, above $200 \mathrm{GHz}$, the dielectric conductivity $\vartheta_{0}(\nu)$ is not shifted. This might be explained by the resonant character of the intermolecular vibrations probed here which are only slightly affected by polarizability. $^{54}$

Besides the individual behaviour of $\varepsilon(\nu)$ and $\vartheta_{0}(\nu)$ the most striking feature of Fig. 5 is their strong overlap over a broad range of frequencies. As a consequence, the total spectrum, $\Sigma_{0}^{*}(\nu)+1$, is of complex nature. In particular, its main peak cannot be solely attributed to either dielectric permittivity or dielectric conductivity but it is essentially created by superposition. This superposition peak is also found in the experimental spectrum shown as the black dashed line in Fig. 6. Its peak position and overall shape agrees with the simulated spectrum for the polarizable system (grey solid line) surprisingly well, whereas the simulated spectrum for the non-polarizable system (grey dashed line) is shifted and differs more in shape. Note that the experimental dielectric spectrum also hints at a further low-frequency component centered at $\sim 0.6 \mathrm{GHz}$, i.e. close to the shoulder in the simulated spectrum. This mode clearly

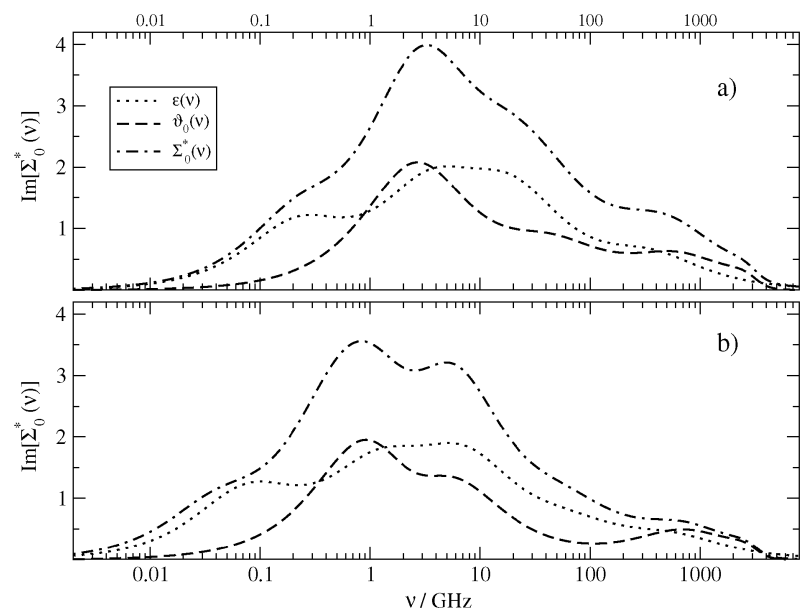

Fig. 5 Imaginary part of the generalized dielectric constant $\Sigma_{0}^{*}(\nu)$ of the polarized (a) and the non-polarized system (b). The dielectric permittivity $\varepsilon(\nu)$ contains rotational and vibrational correlations and the dielectric conductivity $\vartheta_{0}(\nu)$ represents the contribution of collective translational phenomena to the generalized dielectric constant. 


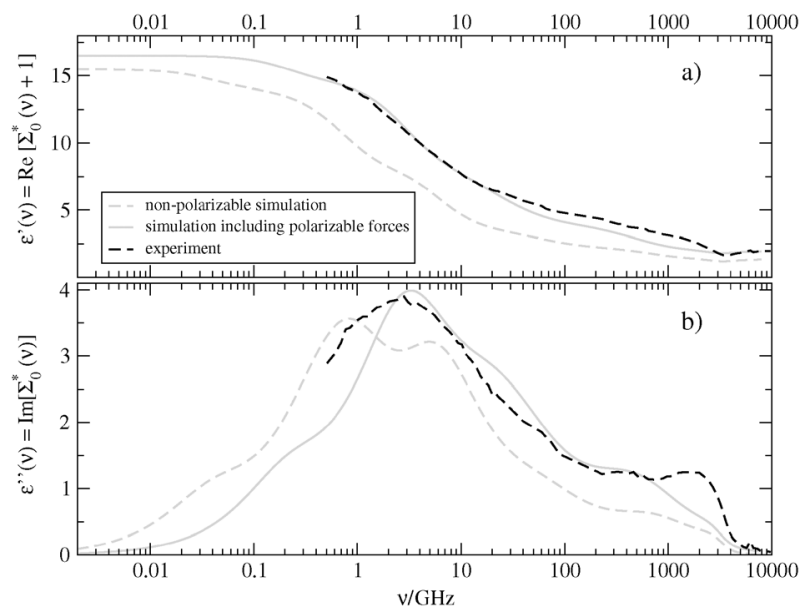

Fig. 6 Real (a) and imaginary part (b) of the "generalized dielectric constant" $\Sigma^{*}(\nu)$ derived from experiment (black dashed line), nonpolarizable (grey dashed) and polarizable (gray solid line) simulation. The high frequency limit $\varepsilon_{\infty}$ equals 2.3 for experiment and 1.9 for simulation, respectively.

shows up in the OKE spectrum of $\mathrm{EMIM}^{+} \mathrm{CF}_{3} \mathrm{SO}_{3}{ }^{-}{ }^{78}$ Such a fair agreement between simulation and experiment was already found for hydrated ILs. ${ }^{21}$ The common feature of hydrated ionic liquids and polarizable systems of ILs is the screening of bare electrostatic forces by an additional "solvent". In the case of hydrated ILs water acts as an explicit solvent. For the polarizable system the induced dipoles behave like an "inner solvent" or lubricant weakening the Coulomb interactions. In ref. 54 a reduction of the Coulomb energy by a factor of $(0.74)^{2}$ was observed without any artificial downscaling of the permanent charges $q_{i \beta}$.

As can be seen from Fig. 6a for the real part of $\Sigma_{0}^{*}(\nu)+1$ the agreement between experimental and computational spectra is almost quantitative below $20 \mathrm{GHz}$ but at higher frequencies the experimental data are systematically larger. In part this is due to the neglected polarizability contribution of the hydrogen atoms in the simulation so that only $\varepsilon_{\infty}=1.9$ is reached for the high-frequency limit, in contrast to the experimental value of 2.3 .

It is possible to calculate $\varepsilon_{\infty}$ directly from atomic polarizabilities, $\alpha_{i \beta}$, with the help of a Claussius-Mossotti type equation ${ }^{54}$

$$
\frac{\varepsilon_{\infty}-1}{\varepsilon_{\infty}+2}=\frac{4 \pi}{3 V} \sum_{i} \sum_{\beta} \alpha_{i \beta}+\mathcal{O}\left(\alpha^{3}\right) .
$$

so that at least for $\omega \rightarrow \infty$ we may estimate the contribution of hydrogen polarizability lacking in our simulations. Based on the atomic polarizabilities of ref. 68 a corrected value of $\varepsilon_{\infty}=2.3$ is thus obtained. Unfortunately, the method of Drude oscillators cannot handle polarizable hydrogens for technical reasons.

As an independent check of the polarizability parameters we have performed MP2-calculations of a single $\mathrm{EMIM}^{+}$and $\mathrm{CF}_{3} \mathrm{SO}_{3}{ }^{-}$molecule with an aug-cc-pVTZ basis set. For the anion the sum $\sum_{\beta} \alpha_{i \beta}$ of the atomic polarizabilities from ref. 68 reproduces the ${ }^{\beta}$ quantum-mechanical result, $\alpha_{i}=8 \AA^{3}$, quite well. In the case of $\mathrm{EMIM}^{+}$the corresponding sum yields $14 \AA^{3}$, whereas the quantum-mechanical calculation predicts a molecular polarizability of $\alpha_{i}=12 \AA^{3}$. The normal mode analysis based on these GAUSSIAN ${ }^{79}$ calculations shows that the lowest intramolecular vibrations of $\mathrm{EMIM}^{+}$have wavenumbers of $\tilde{v} \simeq 48$ and $77 \mathrm{~cm}^{-1}$ whereas a band at $\tilde{\nu} \simeq 67 \mathrm{~cm}^{-1}$ is expected for triflate. The visualization of these normal modes reveals that they are mainly characterized by an internal rotation of the ethyl- and methyl chain and the torsion around the carbon-sulfur bond, respectively. In the scale of Fig. 6 these intramolecular vibrations should show up at frequencies of $\sim 1600$ and $2400 \mathrm{GHz}$. Apparently these modes contribute significantly to the experimental spectrum, which shows a clear peak at $\sim 2400 \mathrm{GHz}$ in the imaginary part (Fig. 6b). Our used force field cannot quantitatively reproduce the experimental GDC at these frequencies although a small shoulder lasts up to $4000 \mathrm{GHz}$. However, it appears that a re-parametrization of the intramolecular torsional barriers after the inclusion of polarizability provides the route for a better reproduction of the experimental spectrum.

\section{Conclusion}

The comparison of the experimental and computed dielectric spectra of $\mathrm{EMIM}^{+} \mathrm{CF}_{3} \mathrm{SO}_{3}{ }^{-}$has shown that the inclusion of polarizable forces is more than a mere numerical improvement which, e.g., increases the diffusion coefficient. In fact, it is essential to account for the correct collective dynamics. The ability of polarizable forces to mimic the response to a changing local environment seems to be constitutive for the description of molecular ILs. The auto-correlation of the induced dipoles of the cations and anions relaxes in a multi-exponential fashion. The overall shape and time constants of these autocorrelation functions resemble those of the permanent collective non-translational dipole moment $\boldsymbol{M}_{D}$. Therefore, the induced dipole moments have to be added to the collective nontranslational dipole moment and thus become part of the dielectric permittivity, $\varepsilon(\nu)$. The collective induced dipole moment of the cations shows a stronger correlation with the permanent non-translational dipole moment of the anions, $\boldsymbol{M}_{D}^{\text {perm- }}{ }^{-}(t)$, than with itself. This indicates a strong anion cage around the cations and may be an explanation for the pronounced low-frequency peak in the OKE spectra of imidazolium $\mathrm{ILs}^{16,78}$ as OKE monitors polarizability fluctuations. The induced dipole moments of the anions anti-correlate with $\boldsymbol{M}_{D}^{\text {perm- }}(t)$. The correlation of the induced dipole moments with the permanent non-translational dipole moment $\boldsymbol{M}_{D}^{\text {perm }}{ }^{+}(t)$ of the cations is much weaker.

Instead of checking a simulation against a set of macroscopic experimental parameters, comparison with the dielectric spectrum offers the possibility to validate a simulation model over a frequency range of several orders of magnitude. We believe that this enables a more systematic validation of force fields. The findings discussed above clearly show that the inclusion of polarization forces has to be done prior to any adjustment of the usual potential parameters, such as LennardJones, partial charges or torsional barriers. Therefore, polarizable force field models augmented by a subsequent adjustment of selected potential parameters open a new field of force field design. The experimentally available dielectric spectra with their wide frequency range offer an excellent opportunity for validation. 


\section{Acknowledgements}

The computational work was performed on the "Vienna Scientific Cluster" (www.zid.tuwien.ac.at/vsc) of the University of Vienna, the Vienna University of Technology, and the University of Natural Resources and Applied Life Science Vienna. We thank for generous allocation of computer time. Thanks also to A. Ortner and M. Walther for providing the THz-TDS data and to D. Turton and K. Wynne for the FIR measurements. This work was supported by Project No. P23494 of the FWF Austrian Science Fund and by the Deutsche Forschungsgemeinschaft within priority program SPP 1191.

\section{References}

1 S. A. Forsyth, J. M. Pringle and D. R. MacFarlane, Aust. J. Chem., 2004, 57, 113.

2 Ionic Liquids in Synthesis, ed. P. Wasserscheid and T. Welton, Wiley-VCH, Weinheim, 2008, 2nd edn, vol. 1.

3 E. W. Castner, Jr and James F. Wishart, J. Chem. Phys., 2010, 132, 120901.

4 H. Weingärtner, Z. Phys. Chem., 2006, 220, 1395.

5 H. Weingärtner, Angew. Chem., Int. Ed., 2008, 47, 654.

6 M.-M. Huang, Y. Jiang, P. Sasisanker, G. D. Driver and H. Weingärtner, J. Chem. Eng. Data, 2011, 56, 1494.

7 U. Schröder, J. D. Wadhawan, R. G. Compton, F. Marken, P. A. Z. Suarez, C. S. Consorti, R. F. de Souza and J. Dupont, New J. Chem., 2000, 24, 1009.

8 J. Dupont, J. Braz. Chem. Soc., 2004, 15, 341.

9 J. N. A. Canongia Lopes and A. A. H. Padua, J. Phys. Chem., 2006, 110, 3330.

10 A. Noda, K. Hayamizu and M. Watanabe, J. Phys. Chem. B, 2001, 105, 4603.

11 H. Cang, J. Li and M. D. Fayer, J. Chem. Phys., 2003, 119, 13017.

12 A. Triolo, O. Russina, V. Arrighi, F. Juranyi, S. Janssen and C. M. Gordon, J. Chem. Phys., 2003, 119, 8549.

13 H. Tokuda, K. Hayamizu, K. Ishii, A. B. H. Susan and M. Watanabe, J. Phys. Chem. B, 2005, 109, 6103.

14 C. Daguenet, P. J. Dyson, I. Krossing, A. Oleinikova, J. Slattery, C. Wakai and H. Weingärtner, J. Phys. Chem. B, 2006, 110, 12682.

15 R. Buchner and G. Hefter, Phys. Chem. Chem. Phys., 2009, 11, 8984.

16 D. A. Turton, J. Hunger, A. Stoppa, G. Hefter, A. Thoman, M. Walther, R. Buchner and K. Wynne, J. Am. Chem. Soc., 2009, 131, 11140.

17 A. Wulf, K. Fumino, R. Ludwig and P. F. Taday, ChemPhysChem, 2010, 11, 349.

18 E. W. Castner and J. F. Wishart, J. Chem. Phys., 2010, 132, 120901.

19 P. A. Hunt, Mol. Simul., 2006, 32, 1.

20 A. Wulf, R. Ludwig, P. Sasisanker and H. Weingärtner, Chem. Phys. Lett., 2007, 439, 323.

21 C. Schröder, J. Hunger, A. Stoppa, R. Buchner and O. Steinhauser, J. Chem. Phys., 2008, 129, 184501.

22 T. Köddermann, K. Fumino, R. Ludwig, J. N. Canongia Lopes and A. A. H. Padua, ChemPhysChem, 2009, 10, 1181.

23 A. Heintz, R. Ludwig and E. Schmidt, Phys. Chem. Chem. Phys., 2011, 13, 3268.

24 C. J. Margulis, H. A. Stern and B. J. Berne, J. Phys. Chem. B, 2002, 106, 12017.

25 J. de Andrade, E. S. Böes and H. Stassen, J. Phys. Chem. B, 2002, 106, 13344.

26 J. N. Canongia Lopes, J. Deschamps and A. A. H. Padua, J. Phys. Chem. B, 2004, 108, 2038.

27 S. U. Lee, J. Jung and Y.-K. Hand, Chem. Phys. Lett., 2005, 406, 332.

28 N. M. Micaelo, A. M. Baptista and C. M. Soares, J. Phys. Chem. B, 2006, 110, 14444.

29 E. J. Maginn, Acc. Chem. Res., 2007, 40, 1200.

30 W. Zhao, H. Eslami, W. L. Cavalcanti and F. Müller-Plathe, Z. Phys. Chem., 2007, 221, 1647.
31 M. S. Kelkar, W. Shi and E. J. Maginn, Ind. Eng. Chem. Res., 2008, 47, 9115.

32 O. Borodin, J. Phys. Chem. B, 2009, 113, 11463.

33 T. I. Morrow and E. J. Maginn, J. Phys. Chem. B, 2002, 106, 12807.

34 J. N. A. Canongia Lopes and A. A. H. Padua, J. Phys. Chem. B, 2006, 110, 7485 .

35 J. de Andrade, E. S. Böes and H. Stassen, J. Phys. Chem. B, 2002, 106, 3546.

36 E. A. Turner, C. C. Pye and R. D. Singer, J. Phys. Chem. A, 2003, $107,2277$.

37 T. G. A. Youngs, M. G. Del Pópolo and J. Kohanoff, J. Phys. Chem. B, 2006, 110, 5697.

38 M. Neumann, Mol. Phys., 1983, 50, 841.

39 M. Neumann, J. Chem. Phys., 1986, 85, 1567.

40 J. M. Caillol, D. Levesque and J. J. Weis, J. Chem. Phys., 1989, 91, 5555.

41 C. Schröder, M. Haberler and O. Steinhauser, J. Chem. Phys., 2008, 128, 134501.

42 C. Schröder and O. Steinhauser, J. Chem. Phys., 2009, 131, 114504.

43 C. Schröder and O. Steinhauser, J. Chem. Phys., 2010, 132, 244109.

44 C. J. F. Böttcher and P. Bordewijk, Theory of electric polarization, Elsevier, Amsterdam, 1978, vol. 2.

45 F. Kremer and A. Schönhals, Broadband Dielectric Spectroscopy, Springer, Berlin, 2002.

46 C. Schröder, C. Wakai, H. Weingärtner and O. Steinhauser, J. Chem. Phys., 2007, 126, 084511.

47 Y. Shim and H. J. Kim, J. Phys. Chem. B, 2008, 112, 11028.

48 X. Song, J. Chem. Phys., 2009, 131, 044503.

49 M. Neumann and O. Steinhauser, Chem. Phys. Lett., 1983, 102, 508.

50 M. Neumann and O. Steinhauser, Chem. Phys. Lett., 1984, 106,563

51 J. M. Caillol, D. Levesque and J. J. Weis, J. Chem. Phys., 1989, 91, 5544.

52 E. I. Izgorodina, M. Forsyth and D. R. MacFarlane, Phys. Chem. Chem. Phys., 2009, 11, 2452.

53 T. Rudas, C. Schröder, S. Boresch and O. Steinhauser, J. Chem. Phys., 2006, 124, 234908.

54 C. Schröder and O. Steinhauser, J. Chem. Phys., 2010, 133, 154511.

55 C. Schröder and O. Steinhauser, in Computational Spectroscopy: Methods, Experiments and Applications, ed. J. Grunenberg, Wiley-VCH, Weinheim, 2010.

56 G. Löffler, H. Schreiber and O. Steinhauser, J. Mol. Biol., 1997, 270, 520.

57 C. Schröder, T. Rudas and O. Steinhauser, J. Chem. Phys., 2006, 125, 244506.

58 H. Yu and W. F. van Gunsteren, Comput. Phys. Commun., 2005, $172,69$.

59 G. Lamoureux and B. Roux, J. Chem. Phys., 2003, 119, 3025.

60 W. Xie, J. Pu, A. D. MacKerell Jr. and J. Gao, J. Chem. Theory Comput., 2007, 3, 1878.

61 B. R. Brooks, C. L. Brooks III, A. D. MacKerell Jr., L. Nilsson, R. J. Petrella, B. Roux, Y. Won, G. Archontis, C. Bartels and S. Boresch, et al., J. Comput. Chem., 2009, 30, 1545.

62 J. N. Canongia Lopes, J. Deschamps and A. A. H. Padua, J. Phys. Chem. B, 2004, 108, 11250.

63 D. A. Case, T. A. Darden, T. E. Cheatham III, C. L. Simmerling, J. Wang, R. E. Duke, R. Luo, K. M. Merz, D. A. Pearlman and M. Crowley, et al., Amber 9, San Francisco, 2006.

64 C. G. Hanke, S. L. Price and R. M. Lynden-Bell, Mol. Phys., 2001, 99, 801.

65 C. Schröder and O. Steinhauser, J. Chem. Phys., 2008, 128, 224503.

66 J. N. Canongia Lopes and A. A. H. Padua, J. Phys. Chem. B, 2004, $108,16893$.

67 J.-P. Ryckaert, G. Ciccotti and H. J. C. Berendsen, J. Comput. Phys., 1977, 23, 327.

68 P. T. van Duijnen and M. Swart, J. Phys. Chem. A, 1998, 102, 2399.

69 V. M. Anisimov, G. Lamoureux, I. V. Vorobyov, N. Huang, B. Roux and A. D. MacKerell, J. Chem. Theory Comput., 2005, 1, 153 . 
70 E. Harder, V. M. Anisimov, T. Whitfield, A. D. MacKerell Jr. and B. Roux, J. Phys. Chem. B, 2008, 112, 3509.

71 E. Harder, V. M. Anisimov, I. V. Vorobyov, P. E. M. Lopes, S. Y. Noskov, A. D. MacKerell Jr. and B. Roux, J. Chem. Theory Comput., 2006, 2, 1587.

72 T. Darden, D. York and L. Pedersen, J. Chem. Phys., 1993, 98, 10089.

73 U. Essmann, L. Perera, M. L. Berkowitz, T. Darden, H. Lee and L. G. Pedersen, J. Chem. Phys., 1995, 103, 8577.

74 J. Barthel, K. Bachhuber, R. Buchner, H. Hetzenauer and M. Kleebauer, Ber. Bunsen-Ges. Phys. Chem., 1991, 95, 853.
75 P. Uhd Jepsen, B. M. Fischer, A. Thoman, H. Helm, J. Y. Suh, R. Lopez and R. F. Haglund, Phys. Rev. B: Condens. Matter Mater. Phys., 2006, 74, 205103.

76 C. Schröder, T. Rudas, G. Neumayr, W. Gansterer and O. Steinhauser, J. Chem. Phys., 2007, 127, 044505.

77 C. Schröder, T. Rudas, G. Neumayr, S. Benkner and O. Steinhauser, J. Chem. Phys., 2007, 127, 234503.

78 T. Sonnleitner, D. Turton, A. Ortner, M. Walther, K. Wynne and R. Buchner, unpublished results.

79 M. J. Frisch, et al., Gaussian 03, Wallingford, CT, 2004. 